\title{
Optics
}

\section{Quasiatomic nanoheterostructures: New model superatom in the alkali - metal atoms}

\author{
Sergey I. Pokutnyi, Petr P. Gorbyk \\ Chuiko Institute of Surface Chemistry, National Academy of Sciences of Ukraine, Kyiv, Ukraine
}

Email address:

pokutnyi_sergey@inbox.ru (S. I. Pokutnyi)

\section{To cite this article:}

Sergey I. Pokutnyi, Petr P. Gorbyk. Quasiatomic Nanoheterostructures: New Model Superatom in the Alkali - Metal Atoms. Optics. Special Issue: Optics and Spectroscopy of the Charge Carriers and Excitons States in Quasi - Zero - Dimensional Nanostructures. Vol. 3, No. 6-1, 2014, pp. 38-41. doi: 10.11648/j.optics.s.2014030601.15

\begin{abstract}
The theory of superatom (or artificial atom) formed from spatially separated electrons and holes (hole moving in the volume of a semiconductor (dielectric) quantum dot and an electron localized on the outer spherical interface between the quantum dot and a dielectric matrix) is developed. Predicted a new hydrogen - artificial atom, which is similar to the new alkali - metal atom.
\end{abstract}

Keywords: Artificial Atoms, Alkali - Metal Atom, Quantum Dots, Superatoms, Separated Electrons and Holes, Quasi-Zero-Dimensional Nanogeterostructures

\section{Introduction}

For the development of mesoscopic physics and chemistry was essential idea superatoms (or artificial atoms) [1-4]. Superatom are nanosized quasi-atomic nanostructure formed from spatially separated electrons and holes (the hole in the volume of the quantum dot (QD) and the electron is localized on the outer spherical quantum dot matrix dielectric interface) [1-4]. This terminology may be correct, given the similarity of the spectra of discrete electronic states of atoms and superatomic and the similarity of their chemical activity [1-4].

In $[1,2]$, in the framework of the modified effective mass method [3], developed the theory of artificial atoms formed from spatially separated electrons and holes (hole moving in the volume of a semiconductor (dielectric) QD and an electron localized on the outer spherical interface between the QD and a dielectric matrix) is developed. The energy spectrum of superatom (exciton of spatially separated electrons and holes) from QD radius $a \geq a$ c (about $4 \mathrm{~nm}$ ) is fully discrete $[1,2,4]$. This is called a hydrogen- superatom. It is localized on the surface of a valence electron QD. The energy spectrum of the superatom consists of a quantumdimension of discrete energy levels in the band gap of the dielectric matrix. Electrons in superatom localized in the vicinity of the nucleus (QD). The electrons moving in welldefined atomic orbitals. Serve as the nucleus of QD containing in its volume semiconductors and insulators. Ionization energy superatoms take large values (of the order of $2.5 \mathrm{eV}$ ), which is almost three orders of magnitude higher than the binding energy of the excitons in semiconductors $[1,2,4]$.

We briefly discuss the possible physical and chemical effects , which are relevant for the results. In our proposed $[1,2,4]$ model of a hydrogen superatom localized on the surface of the QD is a valence electron. In a quasi-atomic structures of the outer valence electron can participate in a variety of physical and chemical processes, similar to the atomic valence electrons in atomic structures. Artificial atoms have the ability to connect to their electron orbitals of electrons $N$ (where $N$ can vary from one to several tens). At the same time, the number of electrons $N$ can take values of the order of a few tens or even surpass the serial numbers of all the known elements of Mendeleev's table $[1,2,4]$. This new effect that allows to attach to the electronic orbitals of artificial atoms $N$ electrons causes a high reactivity, and opens up new possibilities superatoms related to their strong oxidizing properties, increasing the possibility of substantial intensity in photochemical reactions during catalysis and adsorption, as well as their ability to form many new compounds with unique properties (in particular, the quasi-molecule and quasicrystals) [4,5,6]. Therefore, studies aimed at a theoretical prediction of the possible existence of artificial 
new atoms (not listed in the table Mendeleev) and, apparently, in their study of the experimental conditions are very relevant.

Quantum discrete states of the individual atoms of alkali metals are determined by the movement of only one, the outermost valence electron around a symmetric atomic core (containing the nucleus and the remaining electrons) [7]. In the hydrogen superatom formed quantum-energy spectra of discrete energy levels of the valence electron $[1,2,4]$. Thus, the observed similarity of the spectra of discrete electronic states and individual superatoms alkali metal atoms, and also the similarity of their chemical activity [1,2,4-7].

In the present communication, on the basis of analogy spectroscopy of electronic states of artificial atoms and individual alkali metal atoms theoretically predicted a new artificial atom, which is similar to the new alkali metal atom.

\section{The New Model of an Superatom in Quasiatomic Nanoheterostructures}

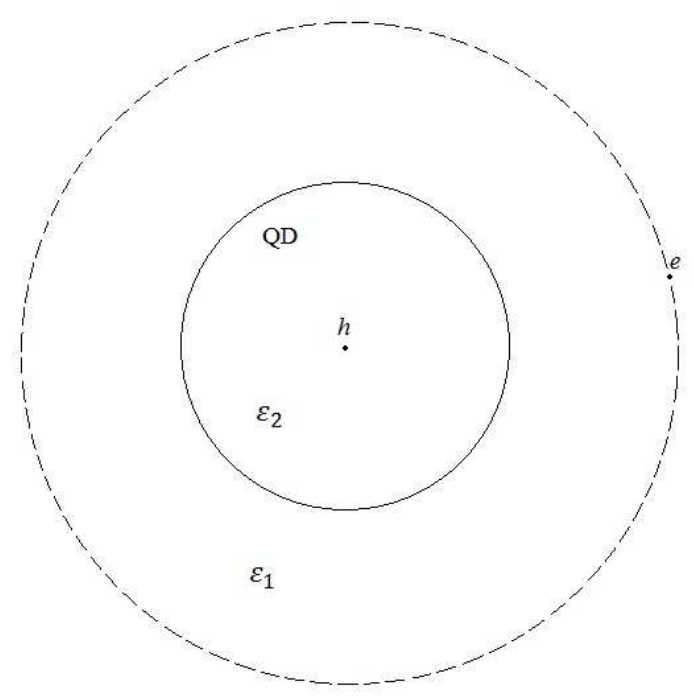

Fig 1. New model of an superatom, which is quasi - zero - dimensional nanosystem consisting of a spherical QD (nucleus superatom) radius a and that includes within its scope semiconductor ( dielectric) with a dielectric constant $\varepsilon_{2}$, surrounded by a dielectric matrix with a dielectric constant $\varepsilon_{1}$. A hole $h$ with the effective mass $m_{h}$ moves in the QD center, while an electron $e$ with the effective mass $m_{e}^{(1)}$ lies in the dielectric matrix.

In $[1,2,4]$ proposed a new model of an superatom, which is quasi - zero - dimensional nanosystem consisting of a spherical QD (nucleus superatom ) radius $a$ and that includes within its scope semiconductor ( dielectric) with a dielectric constant $\varepsilon_{2}$, surrounded by a dielectric matrix with a dielectric constant $\varepsilon_{1}$. A hole $h$ with the effective mass $m_{h}$ moves in the QD volume, while an electron $e$ with the effective mass $m_{e}^{(1)}$ lies in the dielectric matrix. In such nanostructure lowest electronic level is situated in the matrix and humble hole level is the volume QD. For the sake of simplicity, while not losing generality, we can assume that the hole occurs at the QD center (see Fig. 1).
Large shift of the valence band (about $700 \mathrm{meV}$ ) is the localization of holes in the volume QD. Large shift of the conduction band (about $400 \mathrm{meV}$ ) is a potential barrier for electrons (electrons move in the matrix and do not penetrate into the volume QD). Coulomb interaction energy of an electron and a hole, and the energy of the electron polarization interaction with the surface section (QD matrix) (since the permittivity $\varepsilon_{2}$ is far superior to QD permittivity $\varepsilon_{1}$ matrix) cause localization of the electron in the potential well above the surface of QD $[1,2]$.

With increasing radius $a \mathrm{QD}$, so that $a>a_{e x}{ }^{0}$, (where

$$
a_{e x}^{0}=\frac{2 \varepsilon_{1} \varepsilon_{2}}{\varepsilon_{1}+\varepsilon_{2}} \cdot \frac{\hbar^{2}}{\mu_{0} e^{2}},
$$

two-dimensional Bohr radius of the electron $(e$ - electron charge, $\mu_{0}=m_{e}{ }^{(1)} m_{h} /\left(m_{e}{ }^{(1)}+m_{h}\right)-$ reduced mass of the electron-hole pair (of spatially separated electron and hole)), spherical surface section (QD- matrix) transforms into a flat surface section. In this artificial atom electron localized on the surface (QD - matrix) becomes two-dimensional. In this case, the potential energy in the Hamiltonian describing the motion of an electron in superatom, the main contribution to the energy of the Coulomb interaction between an electron and a hole [1,2]:

$$
V_{e h}(r)=-\frac{1}{2}\left(\frac{1}{\varepsilon_{1}}+\frac{1}{\varepsilon_{2}}\right) \frac{e^{2}}{r} .
$$

(where $r$ - distance of the electron from the center QD). Polarization interaction energy of the electron and hole with a spherical surface section (QD - matrix) gives a much smaller contribution to the potential energy of the Hamiltonian. Thus contribute to a first approximation can be neglected $[1,2]$. In this regard, the two-dimensional electron energy spectrum in the artificial atom takes the form [1,2]:

$$
E_{n}=-\frac{R y_{e x}^{0}}{(n+(1 / 2))^{2}}, R y_{e x}^{0}=\frac{\left(\varepsilon_{1}+\varepsilon_{2}\right)^{2}}{4 \varepsilon_{1}^{2} \varepsilon_{2}^{2}} \cdot\left(\frac{\mu_{0}}{m_{0}}\right) R y_{0},
$$

where $n=0,1,2$ - the principal quantum number of the electron, $R y_{0}=13.606 \mathrm{eV}-$ Rydberg constant. The binding energy of the ground state of the two-dimensional electron, according to (3), is given by:

$$
E_{e x}^{0}=-4 R y_{e x}^{0} \text {. }
$$

Under the binding energy (4) refers to the electron ground state energy required for the decay of the bound state of an electron and a hole (in the state with $n=0$ ).

Dependence of the binding energy $E_{e x}(a, \varepsilon)$ of an electron in the ground state superatom (QD containing zinc selenide radius $a$ and surrounded by a matrix of borosilicate glass [8]), obtained in $[1,2]$ by the variational method, it follows that the bound state of an electron occur near spherical interface (QD-matrix), starting with the value of the critical 
radius QD $a \geq a_{c}^{(1)}=3.84 \mathrm{~nm}$. When this hole moves in a volume QD, and the electron is localized on the surface of the spherical section (QD - matrix). In this case, the Coulomb interaction energy $V_{e h}(r)$ (2) between the electron and the hole, and the energy of the polarization interaction of electrons and holes with a spherical surface section (QDmatrix) prevail over the size quantization of the energy of electrons and holes in the artificial atom. Thus, in [1,2] found that the occurrence of superatom has a threshold, and is only possible since the radius of QD KT $a \geq a_{c}^{(1)}=3.84$ $\mathrm{nm}$.

With increasing radius of $a$ QD scan, an increase in the binding energy of the electron in the ground state superatom. In the range of radii $4.0 \leq a \leq 29.8 \mathrm{~nm}$ and the binding energy of the electron in the ground state superatom significantly exceeds (in $(4,1-76,2)$ times) the value of the exciton binding energy $\tilde{E}_{e x}^{0} \approx 21.07 \mathrm{meV}$ in a single crystal of zinc selenide [1,2]. Beginning with $a$ radius QD $a \geq a_{c}^{(2)}=29.8 \mathrm{~nm}$, the energy of the ground state of an electron in superatom asymptotically follow the value $E_{e x}^{0}=-1.5296 \mathrm{eV}$, which characterizes the energy of the ground state of two-dimensional electrons in an artificial atom (4) [1,2].

Effect of significantly increasing the energy of the ground state of an electron in superatom mainly determined by two factors [1,2]: 1) a significant increase in the Coulomb interaction energy $\left|V_{e h}(r)\right|$ (2) electron-hole (the "dielectric enhancement" [9]); 2) the spatial limitation on the quantization volume QD, while with increasing radius of $a \mathrm{QD}$, since the radius of QD $a \geq a_{c}^{(2)}=52 a^{0}{ }_{e x}=29.8 \mathrm{~nm}$ superatomic becomes two-dimensional with a binding energy of the ground state $E_{e x}^{0}$ (4), the value of which is almost two order exceeds the exciton binding energy in a single crystal of zinc selenide. Effect of "dielectric enhancement" due to the fact that when the dielectric constant $\varepsilon_{1}$ of the matrix is much less than the dielectric constant of $\mathrm{QD} \varepsilon_{2}$, an essential role in the interaction between the electron and the hole in the superatom playing field produced by these quasi-particles in a matrix. Thus, the interaction between the electron and the hole in the superatom is significantly larger than in a semiconductor permittivity $\varepsilon_{2}$ [9].

\section{New Model Artificial Atom, which is Similar to a New Single Alkali Metal Atom}

Quantum discrete states of the individual atoms of alkali metals are determined by the movement of only one, the outermost valence electron around a symmetric atomic core (containing the nucleus and the remaining electrons) [7]. At large distances $r$ electron from the nucleus (so that $r>>a 0$, where $a 0=0.053 \mathrm{~nm}$ - the Bohr radius of the electron in a hydrogen atom), the field of the atomic core is described by the Coulomb field [7]:

$$
\mathrm{V}(r)=-\left(Z e^{2} / r\right)
$$

determining the interaction of the valence electron with the atomic core $(\mathrm{Z}$ - serial number of the atom in the periodic table Mendeleev). The energy spectrum of a single atom of an alkali metal hydrogen-described spectrum [7]:

$$
E_{n^{*}}=-\frac{R y^{*}}{\left(n^{*}\right)^{2}}, R y *=Z^{2} R y_{0},
$$

where $n^{*}=(n+y)$ - effective quantum number $(n=1,2$, $3, \ldots$. - the principal quantum number), the amendment $y$ depends on the orbital quantum number $l$. Amendment $y$ in due to the fact that the valence electron moves in the Coulomb field of the atomic core, where the nuclear charge is screened by core electrons. Amendment $y$ correction is determined by comparing the spectrum of (6) with its experimental values. The value of $y<0$, and numerically in the more closer to the atomic core suitable valence electron orbit. The number of possible orbits of the valence electron in a single alkali metal atom such as a hydrogen atom, and [7].

The similarity of the individual series of neutral alkali metal atoms with hydrogen Balmer series suggests that the energy spectra of neutral alkali metal atoms are called valence electron radiation in transitions from higher levels to the level of principal quantum number $n=2$ [7]

In a single atom of an alkali metal valence electron moving in the Coulomb field of the atomic core (5) having the same functional dependence on $r$ as the Coulomb field (2), in which the valence electron in hydrogen-like model of artificial atom. This leads to the fact that the energy spectra of the valence electron in a single atom of an alkali metal (6) and in the artificial atom (3) describes the spectrum of hydrogen-type. At the same time, the number of possible quantum states of valence electron in hydrogenlike artificial atom model is the same as the number of quantum states of discrete valence electron in a single atom of an alkali metal $[1,2,4,7]$.

The Table 1 shows the position of the valence electron energy levels in individual atoms of alkali metals $(\mathrm{K}, \mathrm{Rb}$, Sc) [7] and the new artificial atom $X$, as well as the level shifts of the valence electron $\left(\Delta E_{R b}^{\mathrm{K}}, \Delta E_{S c}^{R b}, \Delta E_{x}^{S c}\right)$ relative to the adjacent level. Assume that the shift of the energy level $E_{x}$ artificial atom X (relative to the energy level $E_{s c}$ of the atom Sc) will be the same as the shift of the energy level $E_{R b}$ of the atom $\mathrm{Rb}$ (relative energy level $E_{s c}$ of the atom $\mathrm{Sc}$ ), (i.e $\Delta E_{x}^{S c}=\Delta E_{S c}^{R b}$ ). Then the level of the valence electron artificial atom will be $E x=-593 \mathrm{meV}$. Using the dependence of the binding energy $E_{e x}(a, \varepsilon)$ of the ground state of an electron in an artificial atom [1,2] (QD containing zinc selenide radius $a$ and surrounded by a matrix of borosilicate glass [8]), we find the radius QD zinc selenide $a 1=5,4 \mathrm{~nm}$, which corresponds to the $E x=-593$ meV. It should be noted that the energy levels of a valence electron in the individual atoms of alkali metals (K, Rb, Sc) [7] and the new artificial atom $X$ are located in the infrared spectrum. 
Table 1. Position of energy levels of the valence electron in some alkali metal atoms $(K, R b, S c)$ and a new artificial atom $X$. Level shifts of the valence electron $\left(\Delta E_{R b}^{K}, \Delta E_{S c}^{R b}, \Delta E_{x}^{S c}\right)$ relative adjacent level.

\begin{tabular}{lll}
\hline $\begin{array}{l}\text { alkali metal } \\
\text { atoms selected }\end{array}$ & $\begin{array}{l}\text { valence electron } \\
\text { energy levels }(\mathbf{m e V})\end{array}$ & $\begin{array}{l}\text { level shifts of the valence } \\
\text { electron }(\mathbf{m e V})\end{array}$ \\
\hline $\mathrm{K}$ & -721.1 & \\
$\mathrm{Rb}$ & -711.2 & 10 \\
$\mathrm{Sc}$ & -652 & 59 \\
$\mathrm{X}$ & -593 & 59 \\
\hline
\end{tabular}

\section{Conclusions}

Thus, we proposed a new model of an artificial atom, which is a quasi-atomic heterostructure consisting of spherical QD (nucleus superatom) radius $a$ and which contains in its scope, zinc selenide, surrounded by a matrix of borosilicate glass (in volume QD moves $h$ hole effective mass $m_{h}, e$ and the electron effective mass $m_{b}{ }^{(1)}$ is located in the matrix), allowed to find a new artificial atom $X$ (absent in the Mendeleev periodic system), which is similar to a new single alkali metal atom. This new artificial atom of valence electron can participate in various physical [1-6, $8,10]$ and chemicals $[4,6]$ processes analogous atomic valence electrons in atomic systems (in particular, alkali metal atoms selected [7]). Such processes are unique due to the new properties of artificial atoms: strong oxidizing properties, increasing the possibility of substantial intensity in photochemical reactions during catalysis and adsorption, as well as their ability to form a plurality of the novel compounds with unique properties (in particular, the quasimolecule and the quasicrystals [4-6] ).

Application of semiconductor nanoheterostructures as the active region nanolasers prevents small exciton binding energy in QD [1-3]. Therefore, studies aimed at finding nanoheterostructures, which would be observed a significant increase in the binding energy of the local electronic states in QDs are relevant [1-3]. Effect of significantly increasing the energy of the electron in a hydrogen superatom $[1,2,4]$ allows to detect experimentally the existence of such superatoms at room temperatures and will stimulate experimental studies nanoheterostructures containing superatoms that can be used as the active region nanolasers working on optical transitions.

\section{References}

[1] Pokutnyi, S.I.: Binding energy of the exciton of a spatially separated electron and hole in quasi - zero - dimensional semiconductor nanosystems. Technical Physics Letters 39, 233 (2013)

[2] Pokutnyi, S.I.: On an exciton with a spatially separated electron and hole in quasi - zero - dimensional semiconductor nanosystems. Semiconductors 47, 791 (2013).

[3] Pokutnyi, S.I.: Exciton states in semiconductor quantum dots in the framework of the modified effective mass method. Semiconductors 41, 1323 (2007).

[4] Pokutnyi, S.I., Gorbyk, P.P.: Superatoms in quasi - zero dimensional nanostructures. J. Appl. Chem. 1, 44 (2013).

[5] Pokutnyi, S.I.: Biexcitons formed from spatially separated electrons and holes in quasi - zero - dimensional semiconductor nanosystems. Semiconductors 47, 1626 (2013).

[6] Pokutnyi, S.I., Gorbyk, P.P.: Quasi - zero - dimensional nanostructures: Excitonic quasimolecules. J. Appl. Chem. 2, 1 (2014).

[7] Frish, S.E.: Optical spectra of atoms. Nauka, Moscow (1963) [in Russian]

[8] Bondar, N.V., Brodyn, M.S.: Spectroscopy of semiconductor quantum dots. Physics E. 42, 1549 (2010).

[9] Keldysh, L.V.: Coulomb interaction between electrons in thin films of semiconductors and semimetals. JETP Lett. 29, 621 (1979).

[10] Pokutnyi, S.I., Gorbyk, A.P.: Local electron states in ellipsoidal nanosystems in homogeneous magnetic field. J. Nanostruct. Chem. 4, 100 (2014). 